\title{
Amount Recovered from T1 to T2
}

National Cancer Institute

\section{Source}

National Cancer Institute. Amount Recovered from T1 to T2. NCI Thesaurus. Code C102359.

The cumulative amount recovered from the specimen type over the interval from $\mathrm{T} 1$ to T2. 